\title{
Direct nucleophilic difluoromethylation of aromatic isoxazoles activated by electron-withdrawing groups using (difluoromethyl) trimethylsilane
}

\author{
Xin Wang ${ }^{1}$, Etsuko Tokunaga ${ }^{2}$, and Norio Shibata ${ }^{1,2 *}$ \\ ${ }^{1}$ Department of Frontier Materials, Nagoya Institute of Technology, Gokiso, Showa-ku, Nagoya, Japan \\ ${ }^{2}$ Department of Nanopharmaceutical Sciences, Nagoya Institute of Technology, Gokiso, Showa-ku, Nagoya, Japan \\ *Corresponding author's e-mail address: nozshiba@nitech.ac.jp \\ Dedication: To Professor Iwao Ojima for his 70th birthday.
}

Published online: 18 December 2014 (version 1)

Cite as: Wang et al., ScienceOpen Research 2015 (DOI: 10.14293/S2199-1006.1.SOR-CHEM.AD1QVW.v1)

Reviewing status: Please note that this article is under continuous review. For the current reviewing status and the latest referee's comments please click here or scan the QR code at the end of this article.

Primary discipline: Chemistry

Keywords: Fluorine, Difluoromethylation, Addition, Isoxazoline, Agrochemical, Insecticide

\section{ABSTRACT}

The activation of aromatic diaryl isoxazoles with strong electron-withdrawing groups, such as the nitro, triflyl, and the phenylsulfonyl groups, at the 4-position has enabled the first regio- and diastereoselective difluoromethylation at the 5-position of isoxazoles by nucleophilic addition using (difluoromethyl) trimethylsilane, $\mathrm{Me}_{3} \mathrm{SiCF}_{2} \mathrm{H}$, to provide difluoromethylated isoxazolines in good yields. Conjugated styryl-4-nitroisoxazoles were also nicely converted into the corresponding $\mathrm{CF}_{2} \mathrm{H}$ adducts with high regio- and excellent diastereoselectivities. Since the trifluoromethylated analogs of the corresponding diaryl-isoxazolines are effective ectoparasiticides, represented by fluralaner, should a series of difluoromethylated isoxazolines be obtained, they would be of great importance as promising drug candidates in this field.

Heterocycles have an extensive history and are present in a wide variety of drugs, most vitamins, many natural products, biomolecules, and biologically active compounds [1-4]. Manmade fluorinated organic compounds have become a remarkable success in the pharmaceutical industry, despite their relatively young history [5-20]. In this context, fluorinated heterocycles have gained attention as new drug candidates over the past few decades in medicine and agro-chemistry [21-28]. Fluorinated and trifluoromethylated compounds have been well targeted in this research area [5-20, 21-28], and difluoromethylated compounds are next [16, 21-28]. The difluoromethyl $\left(\mathrm{CF}_{2} \mathrm{H}\right)$ group is known to be isosteric and isopolar to a hydroxy $(\mathrm{OH})$ and thiol $(\mathrm{SH})$ unit. The $\mathrm{CF}_{2} \mathrm{H}$ group can also act as a more lipophilic hydrogen donor than $\mathrm{OH}$ and $\mathrm{NH}$ groups through hydrogen bonding [31-34]. Thus, the difluoromethylation of biologically active molecules is an effective strategy for the design new candidates of pharmaceuticals and agrochemicals [16].
BRAVECTO ${ }^{\mathrm{TM}}$ (fluralaner) is a highly potent insect and acarid RDL and GluCl inhibitor that was just recently approved in chewable tablets for dogs against fleas and ticks [35]. A systematically large number of research disclosed that 3,5-diaryl-5-(trifluoromethyl)-2-isoxazoline unit $\mathbf{1}$ is a key skeleton for its biological activity [36-38]. Since 2010, our group has also made contributions to this fascinating structure by the direct late-stage trifluoromethylation of aromatic isoxazoles with Ruppert-Prakash reagent (trifluoromethyl) trimethylsilane $\left(\mathrm{Me}_{3} \mathrm{SiCF}_{3}\right)$ [39-40,41], and a fluorinated building block strategy based on the use of inexpensive reagents under organocatalysis with an eye on industrial purposes [36-38]. We are now interested in the synthesis of difluoromethyl analogs of this key structure, i.e., 3,5-diaryl-5-(difluoromethyl)-2-isoxazolines 2. More than 27,000 isoxazolines 1 with a quaternary carbon bearing a $\mathrm{CF}_{3}$ group at the 5-position have been synthesized and patented [42]; however, common structures bearing a $\mathrm{CF}_{2} \mathrm{H}$ group 2 are rare [43] (19 compounds, 4 patents Figure 1).

In this paper, we disclose the first direct difluoromethylation at the 5-position of diary-isoxazoles 3 by nucleophilic addition using (difluoromethyl) trimethylsilane $\left(\mathrm{Me}_{3} \mathrm{SiCF}_{2} \mathrm{H}\right)$ in the presence of tetramethylammonium fluoride at room temperature. A series of diary-isoxazoles $\mathbf{3}$ having a nitro (X = $\left.\mathrm{NO}_{2}\right)$, triflyl $\left(\mathrm{X}=\mathrm{SO}_{2} \mathrm{CF}_{3}\right)$, or phenylsulfonyl $\left(\mathrm{X}=\mathrm{SO}_{2} \mathrm{Ph}\right)$ group at the 4-postion are nicely $\mathrm{CF}_{2} \mathrm{H}$-functionalized under the same mild conditions with good to high diastereoselectivity. Nucleophilic difluoromethylation of 1,6-conjugated styryl4-nitro isoxazoles was also achieved with $\mathrm{Me}_{3} \mathrm{SiCF}_{2} \mathrm{H}$ under the same reaction conditions to provide $\mathrm{CF}_{2} \mathrm{H}$-adducts 4, with high regio- and excellent diastereoselectivities. A wide variety of $\mathrm{CF}_{2} \mathrm{H}$ analogs of agrochemically attractive diarylisoxazolines $\mathbf{2}$ and their styryl analogs $\mathbf{4}$ were synthesized by this method. The nitro group in products $2\left(\mathrm{X}=\mathrm{NO}_{2}\right)$ 


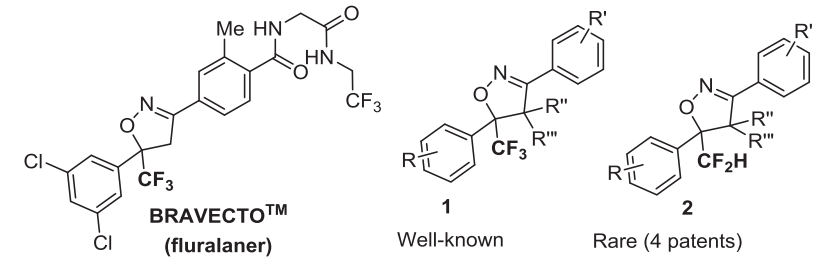

Figure 1. BRAVECTO ${ }^{\mathrm{TM}}$, trifluoromethyl-diaryisoxazolines 1 and their difluoromethyl analogs 2 .

can be removed under radical reaction conditions to afford $2(\mathrm{X}=\mathrm{H})$. The patented examples of this skeleton are synthesized by a so-called building block strategy [44-47]; hence, our method is the first example of the synthesis of 3,5-diaryl5-(difluoromethyl)-2-isoxazolines by a direct difluoromethylation reaction (Figure 2).

In our previous studies, direct trifluoromethylation into the 5-position of isoxazoles was achieved by using the RuppertPrakash reagent, $\mathrm{Me}_{3} \mathrm{SiCF}_{3}$ [39-41]. Therefore, difluoromethylation with $\mathrm{Me}_{3} \mathrm{SiCF}_{2} \mathrm{H}$ instead of $\mathrm{Me}_{3} \mathrm{SiCF}_{3}$ under the same conditions is an ideal extension of this strategy. However, the use of $\mathrm{Me}_{3} \mathrm{SiCF}_{2} \mathrm{H}$ instead of $\mathrm{Me}_{3} \mathrm{SiCF}_{3}$ is not just a simple extension of direct trifluoromethylation, due to the rather inactive character of $\mathrm{Me}_{3} \mathrm{SiCF}_{2} \mathrm{H}$ [48, 49]. According to molecular orbital calculations of (difluoromethyl)- and (trifluoromethyl)fluorotrimethylsilicates reported by Fuchikami et al. [48], the bond order of the $\mathrm{Si}-\mathrm{CF}_{2} \mathrm{H}$ bond (0.436) is significantly higher than that of the $\mathrm{Si}-\mathrm{CF}_{3}$ bond (0.220); eventually, the cleavage of the $\mathrm{Si}-\mathrm{CF}_{2} \mathrm{H}$ bond is more difficult than that of the $\mathrm{Si}_{-} \mathrm{CF}_{3}$ bond. Since Fuchikami's report, difluoromethylsilanes were believed to be useless for nucleophilic difluoromethylation until a recent report emerged from $\mathrm{Hu}$ et al. in 2011. They developed a Lewis base that could activate the nucleophilic difluoromethylation of various aldehydes, ketones, and imines with $\mathrm{Me}_{3} \mathrm{SiCF}_{2} \mathrm{H}$ at room temperature or even at low temperature [49]. Encouraged by their work, combined with the advances of our previous work, we initialized the optimization of the reaction conditions for the difluoromethylation of 4-nitro-3,5-diphenylisoxazole (3a), using $\mathrm{Me}_{3} \mathrm{SiCF}_{2} \mathrm{H}$.

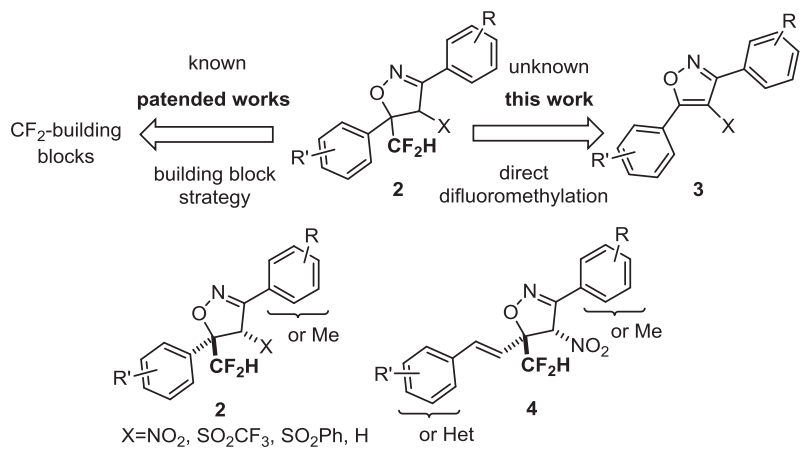

Figure 2. Two strategies for difluoromethylated isoxazolines 2 and newly obtained difluoromethylated isoxazolines 2 and 4 .
We first attempted difluoromethylation under the previous best conditions for trifluoromethylation of $\mathbf{3 a}[39,40]$ or 4triflyl-3,5-diphenylisoxazole (3f) [41], however, the results were not satisfactory (Table 1, entries 1 and 2). There was no reaction in the presence of other basic conditions (entries 3 5). Yield improved to $22-33 \%$ when phase-transfer catalyst 18-crown-6 (1.5 equiv) was added with potassium acetate and potassium fluoride (entries 6 and 7). Interestingly, ammonium salt tetramethylammonium fluoride $\left(\mathrm{Me}_{4} \mathrm{NF}\right)$ could cleave the $\mathrm{Si}-\mathrm{CF}_{2} \mathrm{H}$ bond more efficiently. The reaction was attempted using $\mathrm{Me}_{4} \mathrm{NF}$ instead of a base, which gave the desired product in 53\% yield (entry 8). Extension of the reaction time did not improve product yield (52\%, entry 9). Traces of the desired product were detected when other quaternary ammonium salts replaced $\mathrm{Me}_{4} \mathrm{NF}$ (entries 10,11). No effect on product yield (52\%) was observed with a catalytic amount of cetyltrimethylammonium bromide (entry 12). Solvent screening did not improve the reaction (entries 1317 ), and the best condition was determined to be entry 8 , by treating 3a with $\mathrm{Me}_{3} \mathrm{CF}_{2} \mathrm{H}$ (2.0 equiv) in $\mathrm{N}, \mathrm{N}$-dimethylformide (DMF) in the presence of $\mathrm{Me}_{4} \mathrm{NF}$ at room temperature for $4 \mathrm{~h}$, and desired product 2a was obtained in 53\% yield (entry 8). The stereochemistry of $\mathbf{2 a}$ was tentatively assigned according to comparisons with previous results $[39,40,41]$.

Assigning the best condition as the standard, we examined the scope of substrates $\mathbf{3}$ for our difluoromethlyation reaction

Table 1. Optimization of reaction conditions.

\begin{tabular}{|c|c|c|c|c|}
\hline & $\underbrace{\mathrm{NO}_{2}}_{3 a}$ & $\begin{array}{l}\text { 1) } \mathrm{Me}_{3} \mathrm{SiCF}_{2} \mathrm{H} \text { ( } 2.0 \text { equiv) } \\
\text { base (1.5 equiv) } \\
\text { additive, solvent, rt, } 4 \mathrm{~h} \\
\text { 2) } 1 \mathrm{~N} \mathrm{HCl}, \mathrm{rt}, 30 \mathrm{~min}\end{array}$ & $\begin{array}{r}\mathrm{HF}_{2} \\
-\quad \mathrm{P}\end{array}$ & ${ }_{2 a}{ }^{\mathrm{NO}_{2}}$ \\
\hline Entry & Base & Additive $^{b}$ & Solvent & Yield $(\%)^{a}$ \\
\hline 1 & $\mathrm{NaOAc}$ & $\begin{array}{l}{\left[\mathrm{CH}_{3}\left(\mathrm{CH}_{2}\right)_{15} \mathrm{~N}\left(\mathrm{CH}_{3}\right)_{3}\right] \mathrm{Br}} \\
\quad(30 \mathrm{~mol} \%)\end{array}$ & DMF & 24 \\
\hline 2 & KOAC & - & DMSO & $N R^{b}$ \\
\hline 3 & $t \mathrm{BuOK}$ & - & DMF & $N R^{b}$ \\
\hline 4 & KOAC & - & DMF & $N R^{b}$ \\
\hline 5 & $\mathrm{KOH}$ & - & DMF & $N R^{b}$ \\
\hline 6 & KOAC & 18-crown-6 (1.5 equiv) & DMF & 22 \\
\hline 7 & $\mathrm{KF}$ & 18-crown-6 (1.5 equiv) & DMF & 33 \\
\hline 8 & $\mathrm{Me}_{4} \mathrm{NF}$ & - & DMF & 53 \\
\hline $9^{c}$ & $\mathrm{Me}_{4} \mathrm{NF}$ & - & DMF & 52 \\
\hline 10 & $\mathrm{Et}_{4} \mathrm{NF} \cdot \mathrm{H}_{2} \mathrm{O}$ & - & DMF & trace $^{b}$ \\
\hline 11 & $n \mathrm{Bu}_{4} \mathrm{NF} \cdot \mathrm{H}_{2} \mathrm{O}$ & - & DMF & trace $^{\mathrm{b}}$ \\
\hline 12 & $\mathrm{Me}_{4} \mathrm{NF}$ & $\begin{array}{l}{\left[\mathrm{CH}_{3}\left(\mathrm{CH}_{2}\right)_{15} \mathrm{~N}\left(\mathrm{CH}_{3}\right)_{3}\right] \mathrm{Br}} \\
\quad(30 \mathrm{~mol} \%)\end{array}$ & DMF & 52 \\
\hline 13 & $\mathrm{Me}_{4} \mathrm{NF}$ & - & THF & $\operatorname{trace}^{\mathrm{b}}$ \\
\hline 14 & $\mathrm{Me}_{4} \mathrm{NF}$ & - & NMP & $N R^{b}$ \\
\hline 15 & $\mathrm{Me}_{4} \mathrm{NF}$ & - & DMSO & trace $^{\mathrm{b}}$ \\
\hline 16 & $\mathrm{Me}_{4} \mathrm{NF}$ & - & DMA & 11 \\
\hline 17 & $\mathrm{Me}_{4} \mathrm{NF}$ & - & DMI & $N R^{b}$ \\
\hline
\end{tabular}

${ }^{\mathrm{a}}$ The yield of isolated product.

${ }^{\mathrm{b}}$ Determined by ${ }^{19} \mathrm{~F}$ NMR analysis of the crude reaction mixture.

${ }^{\mathrm{c}}$ Reaction ran for $48 \mathrm{~h}$. 
in order to establish the generality of the process. A series of 3,5-diary-4-nitro-isoxazole 3 with different substituents at aromatic rings, including electron-donating and electron-withdrawing groups, were converted into corresponding difluoromethylated adducts smoothly in moderate yields with good to excellent diastereoselectivities (d.r. = 85:15-97:3, Table 2, $\mathbf{2 a - 2 e )}$. It should be noted that isoxazoles having a different electron-withdrawing group at the 4-position, $\mathrm{SO}_{2} \mathrm{CF}_{3}$ and $\mathrm{SO}_{2} \mathrm{Ph}$, i.e., 3,5-diphenyl-4-(trifluoromethanesulfonyl)isoxazole 3f and 3,5-diphenyl-4-(phenylsulfonyl)isoxazole 3g, were also suitable substrates for this transformation, affording difluoromethylated adducts $\mathbf{2} \mathbf{f}$ and $\mathbf{2 g}$ in moderate yields but rather low diastereoselectivities.

We next investigated the difluoromethylation of 4-nitro-5styrylisoxazoles $\mathbf{5}$. These compounds are flexible building blocks that bear a number of different functionalities [50-66]. Generally, 4-nitro-5-styrylisoxazoles $\mathbf{5}$ have two electrophilic centres, both of which can be attacked by nucleophiles [5066]. Although the addition of carbon nucleophiles at the 4position of 5 is rare, according to our previous work [39, 40], nucleophilic trifluoromethylation to conjugated alkenes with $\mathrm{Me}_{3} \mathrm{SiCF}_{3}$ fundamentally occurs exclusively by a 1,2-addition, and not a 1,4-addition. These results indicate that the addition of an aromatic isoxazole ring at the 5-position is specific to the trifluoromethylation reaction. In this work, we investigated the nucleophilic difluoromethylation of various 4-nitro5-styrylisoxazoles 5 under the same reaction conditions to afford 1,2-addition difluoromethylated compounds as main

Table 2. Stereoselective difluoromethylation of 3,5-diarylisoxazole $\mathbf{3}$ by nucleophilic difluoromethylation. ${ }^{a}$

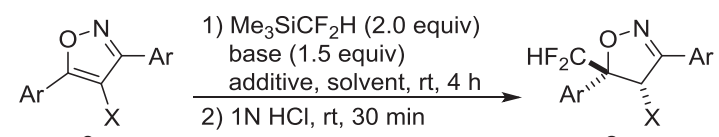

3

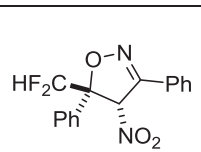

2a: $53 \%$, d.r. $=90: 10$

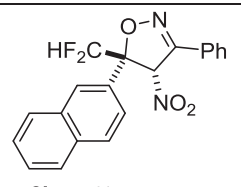

2b: $49 \%$, d.r. $=97: 3$
2

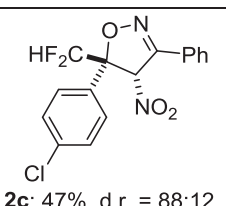

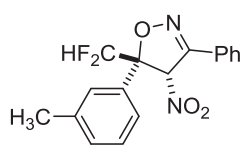

2d: $51 \%$, d.r. $=87: 13$

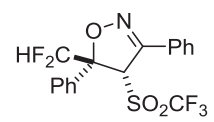

2f: $40 \%$ d.r. $=56: 44$

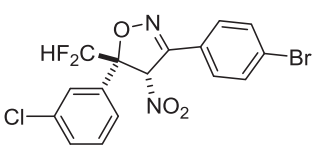

2e: $50 \%$ d.r. $=85: 15$

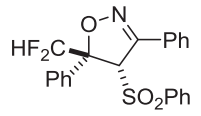

2g: $42 \%$, d.r. $=78: 22$
${ }^{a}$ Yield of the isolated products.
Table 3. Regio- and diastereoselective difluoromethylation of 4-nitro-5-styrylisoxazoles by nucleophilic addition. ${ }^{\text {a }}$
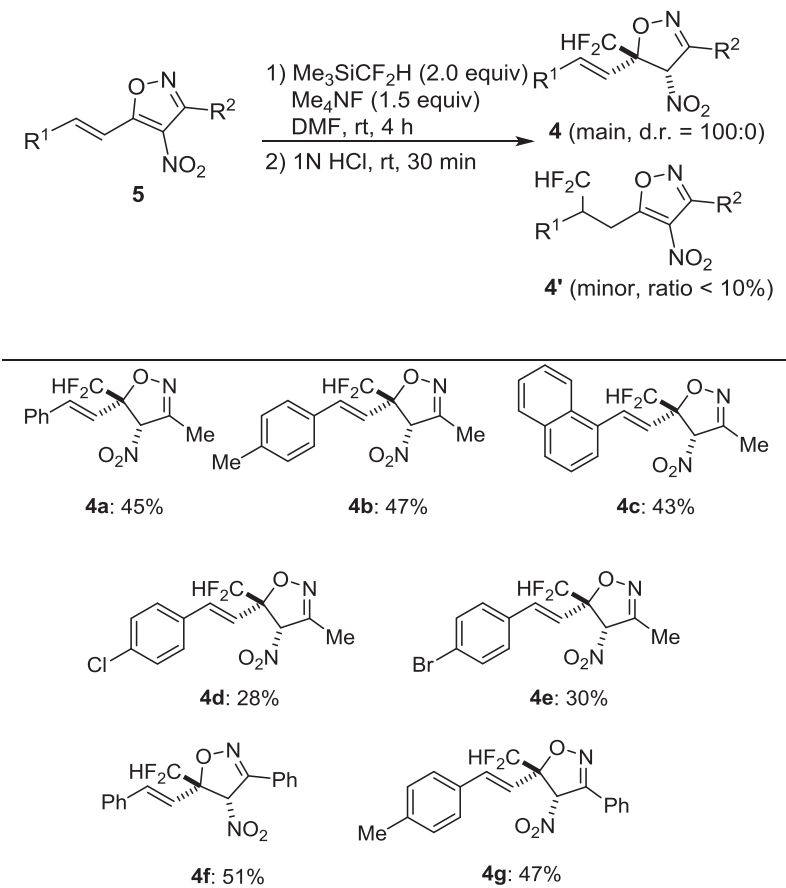

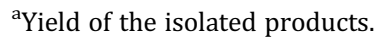

products, while a very small amount of the 1,4-addition of difluoromethylated compound was observed (less than $10 \%$ of all difluoromethylated products) (Table 3). 3-Methyl-5difluoromethyl-5-styrylisoxazoles were obtained with complete diastereoselectivity as single isomers (4a-4e). Moderate yields were obtained when $\mathrm{R}^{1}$ was an electron-rich aromatic ring or a non-substrate benzene ring $\left(\mathbf{4 a - 4 c )}\right.$. When $\mathrm{R}^{1}$ was an electron-poor aromatic ring, lower yields of corresponding products were obtained (4d and 4e). 3-Aryl-5-styrylisoxazoles were next investigated for the difluoromethylation and gave the corresponding products smoothly as single isomers (4f and 4g).

In conclusion, the activation of aromatic isoxazoles with a strong electron-withdrawing group at the 4-position has resulted in the realization of the first diastereoselective difluoromethylation at the 5-position of isoxazoles 3 by nucleophilic addition using $\mathrm{Me}_{3} \mathrm{SiCF}_{2} \mathrm{H}$. Regio- and diastereoselective difluoromethylation by nucleophilic addition was also achieved in the reaction with 1,6-conjugated styryl-4-nitroisoxazoles 4 under the same reaction conditions. Notably, a strong electron-withdrawing group at the

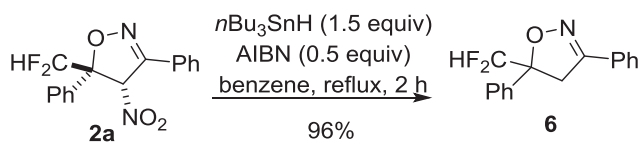

Figure 3. Removal of the $\mathrm{NO}_{2}$ group of $2 \mathrm{a}$ to 6 . 
4-position is essential for this addition. No reaction was observed without the use of non-substituted 3,5-diphenyl isoxazole as substrate under the same reaction conditions. The nitro group of $\mathbf{2 a}$ was easily removed under radical reduction conditions to provide 6 in 96\% yield (Figure 3). Therefore, this method provides a new series of highly functionalized 5-difluoromethyl-2-isoxazoline derivatives that may be attractive candidates for agrochemicals. The biological activities of selected 5-(difluoromethyl)-2-isoxazoline derivatives 2 and asymmetric variants of this method are now under consideration.

\section{EXPERIMENTAL SECTION}

All reagents were used as received from commercial sources, unless specified otherwise, or prepared as described in the literature. Reactions requiring anhydrous conditions were performed in oven-dried glassware under a positive pressure of nitrogen in glove box. Reaction mixtures were stirred magnetically. Solvents were transferred via syringe and were introduced into the reaction vessels though a rubber septum. All of the reactions were monitored by thin-layer chromatography (TLC) carried out on $0.25 \mathrm{~mm}$ Merck silica gel (60F254). The TLC plates were visualized with UV light and 7\% phosphomolybdic acid or $\mathrm{KMnO}_{4}$ in water/heat. Column chromatography was carried out on a column packed with silica gel $60 \mathrm{~N}$ spherical neutral size $63-210 \mu \mathrm{m}$. The ${ }^{1} \mathrm{H}$-NMR $(300$ $\mathrm{MHz}$ ) and ${ }^{19} \mathrm{~F}-\mathrm{NMR}$ (282.3 $\mathrm{MHz}$ ) spectra was recorded on a Varian Mercury 300. The ${ }^{13} \mathrm{C}-\mathrm{NMR}(150.9 \mathrm{MHz})$ was recorded on a Bruker Avance 600. Chemical shifts $(\delta)$ are reported in parts per million and coupling constants $(J)$ are in hertz. Mass spectra were recorded on a SHIMADZU LCMS-2020. All the isoxazole substrates (3 and 5) were prepared according to the literature procedure $[39,40,67]$.

General procedure for the difuoromethylation of 4-nitroisoxazoles: in a flame dried test tube, 4-nitroisoxazole $\mathbf{3}$ or $\mathbf{5}(0.2$ mmol) and $\mathrm{Me}_{4} \mathrm{NF}(0.3 \mathrm{mmol})$ added in glove box. DMF (2.0 $\mathrm{mL})$ and $\mathrm{Me}_{3} \mathrm{SiCF}_{2} \mathrm{H}(0.4 \mathrm{mmol})$ was added in the mixture. After stirring at room temperature for $4.0 \mathrm{~h}, 1 \mathrm{~N} \mathrm{HCl}$ aq. was added to the reaction mixture and stirred for 30 minutes. After dilution with water, the whole reaction mixture was extracted with AcOEt, The combined organic phase was washed successively with water and saturated brine, and then dried over anhydrous $\mathrm{Na}_{2} \mathrm{SO}_{4}$. The solution was filtered and the solvent was removed under reduced pressure. The crude product was further purified by silica gel column chromatography or prepared TLC to give $\mathbf{2 a - g}$ and $\mathbf{4 a - g}$.

5-(difluoromethyl)-4-nitro-3,5-diphenyl-4,5-dihydroisoxazole (2a): Following the general procedure, 2a was isolated (33.7 mg, $53 \%)$ as a yellow solid. ${ }^{1} \mathbf{H}$ NMR $\left(\mathrm{CDCl}_{3}, 300 \mathrm{MHz}\right) \delta$ $5.95(\mathrm{t}, J=54.9 \mathrm{~Hz}, 1 \mathrm{H}), 6.62(\mathrm{~s}, 1 \mathrm{H}), 7.43-7.75(\mathrm{~m}, 10 \mathrm{H}) ;{ }^{19} \mathbf{F}$ NMR (282 MHz, $\mathbf{C D C l}_{3}$ ) $\delta-128.52$ (ddd, $J=505.6 \mathrm{~Hz}, 282.0$ $\mathrm{Hz}, 53.6 \mathrm{~Hz}, 2 \mathrm{~F}) ;{ }^{13} \mathbf{C}$ NMR (150.9 $\left.\mathbf{M H z}, \mathbf{C D C l}_{3}\right) \delta$ 152.86, $131.94,130.36,129.57,129.12,126.80,126.53,125.97$, $113.82(\mathrm{t}, J=254.6 \mathrm{~Hz}), 92.90,92.05(\mathrm{t}, J=21.4 \mathrm{~Hz})$; MS (ESI, $\boldsymbol{m} / \mathbf{z}) 317[\mathrm{M}-\mathrm{H}]^{-}$. 5-(difluoromethyl)-5-(naphthalen-2-yl)-4-nitro-3-phenyl-4,5dihydroisoxazole (2b): following the general procedure, $\mathbf{2 b}$ was isolated (36.1 mg, 49\%) as a yellow solid. ${ }^{1} \mathbf{H}$ NMR $\left(\mathrm{CDCl}_{3}, 300 \mathrm{MHz}\right) \delta 6.02(\mathrm{t}, J=55.5 \mathrm{~Hz}, 1 \mathrm{H}), 6.70(\mathrm{~s}, 1 \mathrm{H})$, 7.44-8.15 (m, 12H); ${ }^{19}$ F NMR (282 $\mathbf{M H z}, \mathbf{C D C l}_{3}$ ) $\delta-128.10$ (ddd, $J=515.5 \mathrm{~Hz}, 283.1 \mathrm{~Hz}, 54.4 \mathrm{~Hz}, 2 \mathrm{~F}) ;{ }^{13} \mathrm{C}$ NMR (150.9 $\left.\mathbf{M H z}, \mathbf{C D C l}_{3}\right) \delta 152.98,133.78,132.89,131.97,129.59$, $129.07,128.82,127.82,127.71,127.08,126.83,125.98$, $122.80,113.99(\mathrm{t}, J=239.6 \mathrm{~Hz}), 92.98,92.255(\mathrm{t}, J=21.0$ $\mathrm{Hz}$ ); MS (ESI, $\boldsymbol{m} / \mathbf{z}) 367[\mathrm{M}-\mathrm{H}]^{-}$.

5-(4-chlorophenyl)-5-(difluoromethyl)-4-nitro-3-phenyl-4,5dihydroisoxazole (2c): following the general procedure, 2c was isolated (33.1 mg, 47\%) as a yellow solid. ${ }^{\mathbf{1}} \mathbf{H}$ NMR $\left(\mathbf{C D C l}_{3}, \mathbf{3 0 0} \mathbf{M H z}\right) \delta 5.92(\mathrm{t}, J=54.9 \mathrm{~Hz}, 1 \mathrm{H}), 6.58(\mathrm{~s}, 1 \mathrm{H})$, 7.40-7.73 (m, 9H); ${ }^{19}$ F NMR (282 $\mathbf{~ M H z , ~} \mathbf{C D C l}_{3}$ ) $\delta-128.36$ (ddd, $J=498.9 \mathrm{~Hz}, 284.0 \mathrm{~Hz}, 53.6 \mathrm{~Hz}, 2 \mathrm{~F}) ;{ }^{13} \mathrm{C}$ NMR (150.9 MHz, $\left.\mathbf{C D C l}_{3}\right) \delta 152.88,136.85,132.09,129.62,129.44$, 128.05, 126.81, 125.76, $113.53(\mathrm{t}, J=254.9 \mathrm{~Hz}), 92.90,91.64$ (t, $J=20.8 \mathrm{~Hz}$; MS (ESI, $\boldsymbol{m} / \mathbf{z}) 351[\mathrm{M}-\mathrm{H}]^{-}$.

5-(difluoromethyl)-4-nitro-3-phenyl-5-(m-tolyl)-4,5-dihydroisoxazole (2d): following the general procedure, $\mathbf{2 d}$ was isolated (33.9 mg, 51\%) as a yellow oil. ${ }^{\mathbf{1}} \mathbf{H}$ NMR $\left(\mathbf{C D C l}_{3}, \mathbf{3 0 0} \mathbf{~ M H z}\right) \delta$ $2.38(\mathrm{~s}, 3 \mathrm{H}), 5.93(\mathrm{t}, J=55.5 \mathrm{~Hz}, 1 \mathrm{H}), 6.58(\mathrm{~s}, 1 \mathrm{H}), 7.22-7.74$ (m, 9H); ${ }^{19}$ F NMR (282 MHz, $\mathbf{C D C l}_{3}$ ) $\delta-128.53$ (ddd, $J=$ $508.4 \mathrm{~Hz}, 282.0 \mathrm{~Hz}, 54.4 \mathrm{~Hz}, 2 \mathrm{~F}) ;{ }^{13} \mathrm{C}$ NMR (150.9 MHz, $\left.\mathbf{C D C l}_{3}\right) \delta 152.85,138.99,131.90,131.11,129.56,128.96$, $126.79,126.02,123.50,113.89(\mathrm{t}, J=255.2 \mathrm{~Hz}), 92.89,92.09$ $(\mathrm{t}, J=21.3 \mathrm{~Hz}$ ), 21.67; MS (ESI, $\boldsymbol{m} / \mathbf{z}) 331[\mathrm{M}-\mathrm{H}]^{-}$.

3-(4-bromophenyl)-5-(3-chlorophenyl)-5-(difluoromethyl)-4nitro-4,5-dihydroisoxazole (2e): following the general procedure, 2e was isolated (43.2 $\mathrm{mg}, 50 \%$ ) as a white solid. ${ }^{\mathbf{1}} \mathbf{H}$ NMR ( $\left.\mathrm{CDCl}_{3}, 300 \mathrm{MHz}\right) \delta 5.94(\mathrm{t}, J=54.3 \mathrm{~Hz}, 1 \mathrm{H}), 6.57(\mathrm{~s}$, 1H), 7.35-7.68 (m, 8H); ${ }^{19}$ F NMR (282 $\left.\mathbf{~ M H z , ~} \mathbf{C D C l}_{3}\right) \delta-$ 128.53 (ddd, $J=597.6 \mathrm{~Hz}, 284.0 \mathrm{~Hz}, 54.4 \mathrm{~Hz}, 2 \mathrm{~F}$ ); ${ }^{13} \mathbf{C}$ NMR (150.9 $\left.\mathbf{M H z}, \mathbf{C D C l}_{3}\right) \delta 152.17,135.41,132.94,130.80$, $130.44,128.14,126.79,124.62,113.43(\mathrm{t}, J=254.9 \mathrm{~Hz})$, 92.61, 91.69 (t, $J=21.4 \mathrm{~Hz}$ ); MS (ESI, $\mathbf{m} / \mathbf{z}) 431[\mathrm{M}-\mathrm{H}]^{-}$.

5-(difluoromethyl)-3,5-diphenyl-4-((trifluoromethyl)sulfonyl)4,5-dihydroisoxazole (2f): following the general procedure, $2 \mathbf{f}$ was isolated (32.4 mg, 40\%) as a white solid. ${ }^{\mathbf{1}} \mathbf{H}$ NMR $\left(\mathrm{CDCl}_{3}, 300 \mathrm{MHz}\right) \delta 5.53(\mathrm{~s}, 0.44 \mathrm{H}), 5.63(\mathrm{~s}, 0.56 \mathrm{H}), 5.87(\mathrm{t}, J$ $=55.8 \mathrm{~Hz}, 0.56 \mathrm{H}), 6.85(\mathrm{t}, J=55.2 \mathrm{~Hz}, 0.44 \mathrm{H}), 7.38-7.76(\mathrm{~m}$, 10H); ${ }^{19}$ F NMR (282 MHz, $\mathbf{C D C l}_{3}$ ) $\delta-73.03,-73.55,-123.00$ (ddd, $J=900.4 \mathrm{~Hz}, 295.0 \mathrm{~Hz}, 53.3 \mathrm{~Hz}, 2 \mathrm{~F}$ ), -127.18 (ddd, $J=$ $384.9 \mathrm{~Hz}, 280.9 \mathrm{~Hz}, 55.3 \mathrm{~Hz}, 2 \mathrm{~F}$ ); MS (ESI, $\boldsymbol{m} / \mathbf{z}$ ) $404[\mathrm{M}-\mathrm{H}]^{-}$. ${ }^{13} \mathrm{C}$ NMR is to complicated to analyze.

5-(difluoromethyl)-3,5-diphenyl-4-(phenylsulfonyl)-4,5-dihydroisoxazole $(\mathbf{2 g})$ : following the general procedure, $\mathbf{2 g}$ was isolated (34.7 $\mathrm{mg}, 42 \%)$ as a white solid. ${ }^{\mathbf{1}} \mathbf{H}$ NMR $\left(\mathbf{C D C l}_{3}\right.$, $300 \mathrm{MHz}) \delta 5.52(\mathrm{t}, J=51.0 \mathrm{~Hz}, 0.78 \mathrm{H}), 5.70(\mathrm{t}, J=55.2 \mathrm{~Hz}$,

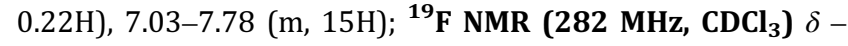
121.86 (ddd, $J=1305.4 \mathrm{~Hz}, 298.9 \mathrm{~Hz}, 53.3 \mathrm{~Hz}, 2 \mathrm{~F}$ ), -128.29 (ddd, $J=480.0 \mathrm{~Hz}, 280.0 \mathrm{~Hz}, 55.3 \mathrm{~Hz}, 2 \mathrm{~F}) ;{ }^{13} \mathrm{C}$ NMR (150.9 MHz, $\left.\mathbf{C D C l}_{3}\right) \delta 155.96,137.15,134.28,131.13,129.62$, 
$129.10,128.85,128.73,128.20,128.01,127.99,114.16(\mathrm{t}, J=$ $255.5 \mathrm{~Hz}$ ), $90.64(\mathrm{t}, J=20.4 \mathrm{~Hz}$ ), 72.36; MS (ESI, $\boldsymbol{m} / \mathbf{z}$ ) 412 $[\mathrm{M}-\mathrm{H}]^{-}$.

5-(difluoromethyl)-3-methyl-4-nitro-5-styryl-4,5-dihydroisoxazole (4a): following the general procedure, 4a was isolated (25.4 $\mathrm{mg}, 45 \%)$ as a yellow oil. ${ }^{\mathbf{1}} \mathbf{H} \mathbf{~ N M R}\left(\mathbf{C D C l}_{3}, \mathbf{3 0 0} \mathbf{~ M H z}\right)$ $\delta 2.16(\mathrm{~s}, 3 \mathrm{H}), 5.85(\mathrm{t}, J=55.8 \mathrm{~Hz}, 1 \mathrm{H}), 5.89(\mathrm{~s}, 1 \mathrm{H}), 6.56(\mathrm{dd}$, $J=292.8 \mathrm{~Hz}, 16.2 \mathrm{~Hz}, 2 \mathrm{H}), 7.34-7.36(\mathrm{~m}, 5 \mathrm{H}) ;{ }^{19}$ F NMR (282 MHz, $\mathbf{C D C l}_{3}$ ) $\delta-130.72$ (ddd, $J=833.3 \mathrm{~Hz}, 285.1 \mathrm{~Hz}, 54.4 \mathrm{~Hz}$ 2F); ${ }^{13} \mathbf{C}$ NMR (150.9 MHz, $\mathbf{C D C l}_{3}$ ) $\delta$ 149.89, 137.93, 134.75, 129.47, 128.92, 127.39, 114.58, 113.72 (t, $J=256.5 \mathrm{~Hz}), 94.48$, $89.22(\mathrm{t}, J=23.2 \mathrm{~Hz}), 12.05$; MS (ESI, $\boldsymbol{m} / \mathbf{z}) 281\left[^{[\mathrm{M}-\mathrm{H}]^{-}}\right.$.

5-(difluoromethyl)-3-methyl-5-(4-methylstyryl)-4-nitro-4,5dihydroisoxazole (4b): Following the general procedure, $4 \mathbf{b}$ was isolated (27.8 mg, 47\%) as a white solid. ${ }^{\mathbf{1}} \mathbf{H}$ NMR $\left(\mathbf{C D C l}_{3}, 300 \mathrm{MHz}\right) \delta 2.35(\mathrm{~s}, 3 \mathrm{H}), 5.92(\mathrm{t}, J=55.8 \mathrm{~Hz}, 1 \mathrm{H})$, 6.40 (s, 1H), 6.61 (dd, $J=295.2 \mathrm{~Hz}, 15.6 \mathrm{~Hz}, 2 \mathrm{H}$ ), 7.14-7.70 (m, 4H); ${ }^{19}$ F NMR (282 $\mathbf{M H z}, \mathbf{C D C l}_{3}$ ) $\delta-130.29$ (ddd, $J=$ $803.4 \mathrm{~Hz}, 285.1 \mathrm{~Hz}, 53.3 \mathrm{~Hz}, 2 \mathrm{~F}$ ); ${ }^{13} \mathrm{C}$ NMR (150.9 MHz, $\left.\mathbf{C D C l}_{3}\right) \delta 149.86,139.63,137.76,132.00,129.60,127.31$, $113.46(\mathrm{t}, J=256.5 \mathrm{~Hz}), 113.39,112.08,94.46,89.28(\mathrm{t}, J=$ $22.9 \mathrm{~Hz}), 21.46,12.05$; MS (ESI, $\boldsymbol{m} / \mathbf{z}) 295[\mathrm{M}-\mathrm{H}]^{-}$.

5-(difluoromethyl)-3-methyl-5-(2-(naphthalen-1-yl)vinyl)-4nitro-4,5-dihydroisoxazole (4c): Following the general procedure, 4e was isolated (28.6 mg, 43\%) as a yellow oil. ${ }^{\mathbf{1}} \mathbf{H}$ NMR ( CDCl $\left._{3}, 300 \mathrm{MHz}\right) \delta 2.20(\mathrm{~s}, 3 \mathrm{H}), 5.95(\mathrm{t}, J=58.5 \mathrm{~Hz}$, 1H), $6.12(\mathrm{~s}, 1 \mathrm{H}), 7.44-8.02(\mathrm{~m}, 9 \mathrm{H}) ;{ }^{19} \mathrm{~F}$ NMR (282 MHz, $\mathbf{C D C l}_{3}$ ) $\delta-130.79$ (ddd, $J=866.0 \mathrm{~Hz}, 285.9 \mathrm{~Hz}, 54.4 \mathrm{~Hz}, 2 \mathrm{~F}$ ); ${ }^{13}$ C NMR (150.9 $\mathbf{M H z}, \mathbf{C D C l}_{3}$ ) $\delta$ 149.94, 135.92, 133.60, $132.77,131.20,129.71,128.70,126.81,126.30,125.61$, 124.79, 123.69, 117.87, $113.70(\mathrm{t}, J=255.9 \mathrm{~Hz}), 94.64,89.33$ $(\mathrm{t}, J=23.2 \mathrm{~Hz}), 12.08$; MS (ESI, $\boldsymbol{m} / \mathbf{z}) 315[\mathrm{M}-\mathrm{H}]^{-}$

5-(4-chlorostyryl)-5-(difluoromethyl)-3-methyl-4-nitro-4,5dihydroisoxazole (4d): following the general procedure, $4 \mathbf{d}$ was isolated (17.7 $\mathrm{mg}, 28 \%)$ as a yellow oil. ${ }^{\mathbf{1}} \mathbf{H}$ NMR $\left(\mathbf{C D C l}_{3}\right.$, $300 \mathrm{MHz}) \delta 2.16(\mathrm{~s}, 3 \mathrm{H}), 5.84(\mathrm{t}, J=54.3 \mathrm{~Hz}, 1 \mathrm{H}), 5.89(\mathrm{~s}$, $1 \mathrm{H}), 6.53(\mathrm{dd}, J=285.6 \mathrm{~Hz}, 15.9 \mathrm{~Hz}, 2 \mathrm{H}), 7.30-7.38(\mathrm{~m}, 4 \mathrm{H})$; ${ }^{19}$ F NMR (282 MHz, $\mathbf{C D C l}_{3}$ ) $\delta-130.67$ (ddd, $J=843.2 \mathrm{~Hz}$, $285.9 \mathrm{~Hz}, 54.4 \mathrm{~Hz}, 2 \mathrm{~F}) ;{ }^{13} \mathbf{C}$ NMR (150.9 $\mathbf{M H z} \mathbf{C D C l}_{3}$ ) $\delta$ 149.92, 136.68, 135.36, 133.21, 129.16, 128.60, 115.26, $113.56(\mathrm{t}, J=252.9 \mathrm{~Hz}), 94.48,89.10(\mathrm{t}, J=23.2 \mathrm{~Hz}), 12.03$; MS (ESI, $\boldsymbol{m} / \mathbf{z}) 315[\mathrm{M}-\mathrm{H}]^{-}$.

5-(4-bromostyryl)-5-(difluoromethyl)-3-methyl-4-nitro-4,5dihydroisoxazole (4e): following the general procedure, $\mathbf{4 e}$ was isolated (21.7 $\mathrm{mg}, 30 \%$ ) as a yellow solid. ${ }^{\mathbf{1}} \mathbf{H}$ NMR $\left(\mathbf{C D C l}_{3}, 300 \mathrm{MHz}\right) \delta 2.17(\mathrm{~s}, 3 \mathrm{H}), 5.84(\mathrm{t}, J=55.2 \mathrm{~Hz}, 1 \mathrm{H})$, 5.89 (s, 1H), 6.53 (dd, $J=275.7 \mathrm{~Hz}, 16.2 \mathrm{~Hz}, 2 \mathrm{H}$ ), 7.22-7.48 $(\mathrm{m}, 4 \mathrm{H}) ;{ }^{19} \mathbf{F}$ NMR (282 $\left.\mathbf{M H z}, \mathbf{C D C l}_{3}\right) \delta-130.69$ (ddd, $J=$ $855.9 \mathrm{~Hz}, 285.9 \mathrm{~Hz}, 54.4 \mathrm{~Hz}, 2 \mathrm{~F}$ ); ${ }^{13} \mathrm{C}$ NMR (150.9 MHz, $\left.\mathbf{C D C l}_{3}\right) \delta 149.92,136.76,133.64,132.12,128.85,123.61$, $115.38,113.54(\mathrm{t}, J=254.0 \mathrm{~Hz}), 94.48,89.11(\mathrm{t}, J=23.1 \mathrm{~Hz})$, 12.03; MS (ESI, $\boldsymbol{m} / \mathbf{z}) 359$ [M-H] $]^{-}$.

5-(difluoromethyl)-4-nitro-3-phenyl-5-styryl-4,5-dihydroisoxazole (4f): following the general procedure, $4 \mathbf{f}$ was isolated
(35.1 mg, 51\%) as a white solid. ${ }^{\mathbf{1}} \mathbf{H}$ NMR $\left(\mathbf{C D C l}_{3}, \mathbf{3 0 0} \mathbf{~ M H z}\right)$ $\delta 5.92(\mathrm{t}, J=56.1 \mathrm{~Hz}, 1 \mathrm{H}), 6.42(\mathrm{~s}, 1 \mathrm{H}), 6.66(\mathrm{dd}, J=290.1 \mathrm{~Hz}$, $16.2 \mathrm{~Hz}, 2 \mathrm{H}), 7.33-7.71(\mathrm{~m}, 4 \mathrm{H}) ;{ }^{19} \mathbf{F}$ NMR (282 $\mathbf{M H z}, \mathbf{C D C l}_{3}$ ) $\delta-130.24$ (ddd, $J=813.6 \mathrm{~Hz}, 285.1 \mathrm{~Hz}, 53.4 \mathrm{~Hz}, 2 \mathrm{~F}$ ); ${ }^{13} \mathrm{C}$ NMR (150.9 MHz, $\mathbf{C D C l}_{3}$ ) $\delta$ 152.34, 138.44, 134.71, 131.84, $129.56,129.53,128.94,127.42,126.76,126.23,114.11$, $113.70(\mathrm{t}, J=253.1 \mathrm{~Hz}), 92.18,90.47(\mathrm{t}, J=21.9 \mathrm{~Hz}) ; \mathbf{M S}$ (ESI, $\mathbf{m} / \mathbf{z}) 343[\mathrm{M}-\mathrm{H}]^{-}$.

5-(difluoromethyl)-5-(4-methylstyryl)-4-nitro-3-phenyl-4,5dihydroisoxazole (4g): following the general procedure, $\mathbf{4 g}$ was isolated (33.7 $\mathrm{mg}, 47 \%$ ) as a white solid. ${ }^{\mathbf{1}} \mathbf{H}$ NMR $\left(\mathbf{C D C l}_{3}, 300 \mathrm{MHz}\right) \delta 5.92(\mathrm{t}, J=56.1 \mathrm{~Hz}, 1 \mathrm{H}), 6.42(\mathrm{~s}, 1 \mathrm{H})$, $6.66(\mathrm{dd}, J=290.1 \mathrm{~Hz}, 16.2 \mathrm{~Hz}, 2 \mathrm{H}), 7.33-7.71(\mathrm{~m}, 4 \mathrm{H}) ;{ }^{19} \mathbf{F}$ NMR (282 MHz, $\mathbf{C D C l}_{3}$ ) $\delta-130.28$ (ddd, $J=802.6 \mathrm{~Hz}, 285.1$ $\mathrm{Hz}, 54.4 \mathrm{~Hz}, 2 \mathrm{~F}) ;{ }^{13} \mathbf{C}$ NMR (150.9 $\mathbf{M H z} \mathbf{C D C l}_{3}$ ) $\delta$ 152.32, $139.72,138.27,131.97,131.80,129.61,129.51,127.35$, 126.74, 126.28, $113.76(\mathrm{t}, J=257.4 \mathrm{~Hz}), 112.92,92.17,90.54$ (t, $J=21.3 \mathrm{~Hz}$ ), 21.46; MS (ESI, $\boldsymbol{m} / \mathbf{z}) 357[\mathrm{M}-\mathrm{H}]^{-}$.

\section{Procedure for denitration of $\mathbf{2 a}$}

To a stirred solution of $2 \mathrm{a}$ (31.8 $\mathrm{mg}, 0.10 \mathrm{mmol}$ ) in benzene $(2.0 \mathrm{~mL})$ were successively added AIBN $(8.2 \mathrm{mg}, 0.050 \mathrm{mmol}$, 0.5 equiv) and $n \mathrm{Bu}_{3} \mathrm{SnH}(40.4 \mu \mathrm{L}, 0.150 \mathrm{mmol}, 1.5$ equiv), and the whole mixture was heated under reflex for $2 \mathrm{~h}$. After cooling down to room temperature, the solution was evaporated under reduced pressure, and the residue was purified by column chromatography on silica gel ( $n$-hexane/ethyl acetate $=$ 95/5) to give 6 (26.2 $\mathrm{mg}, 96 \%)$ as a white solid. ${ }^{\mathbf{1}} \mathbf{H}$ NMR $\left.\mathbf{( C D C l}_{3}, \mathbf{3 0 0} \mathbf{M H z}\right) \delta 3.82(\mathrm{dd}, J=124.8 \mathrm{~Hz}, 16.8 \mathrm{~Hz}, 2 \mathrm{H}), 5.91$ $(\mathrm{t}, J=55.2 \mathrm{~Hz}, 1 \mathrm{H}), 7.36-7.70(\mathrm{~m}, 10 \mathrm{H}) ;{ }^{19} \mathbf{F}$ NMR (282 MHz, $\mathbf{C D C l}_{3}$ ) $\delta-129.10(\mathrm{ddd}, J=371.1 \mathrm{~Hz}, 281.2 \mathrm{~Hz}, 55.3 \mathrm{~Hz}, 2 \mathrm{~F}$ ); ${ }^{13}$ C NMR (150.9 MHz, $\mathbf{C D C l}_{3}$ ) $\delta 156.46,137.52$ (d, $J=2.8$ $\mathrm{Hz}), 130.73,129.04,128.95,128.83,128.73,126.94,126.48$, $114.63(\mathrm{t}, J=251.6 \mathrm{~Hz}), 88.46(\mathrm{t}, J=22.5 \mathrm{~Hz}), 41.77$; MS (ESI, $m / z) 274[\mathrm{M}+\mathrm{H}]^{+}$.

\section{ACKNOWLEDGEMENTS}

This research was financially supported in part by the Platform for Drug Discovery, Informatics, and Structural Life Science from MEXT Japan, the Advanced Catalytic Transformation (ACT-C) from the Japan Science and Technology (JST) Agency, Scientific Research (B) (25288045) and Exploratory Research (25670055) from JSPS, and Kobayashi International Scholarship Foundation. We also thank Professor Jinbo $\mathrm{Hu}$ for a gift of $\mathrm{Me}_{3} \mathrm{SiCF}_{2} \mathrm{H}$.

\section{REFERENCES}

[1] Eicher T, Hauptmann S, Suschitzky H. The chemistry of heterocycles: structure, reactions, syntheses and applications. Hoboken (NY): Wiley; 2003.

[2] Katritzky AR. Comprehensive heterocyclic chemistry III. 1st ed. Amsterdam (the Netherlands): Elsevier; 2008.

[3] Katritzky AR, Ramsden CA, Joule JA, Zhdankin VV. Handbook of heterocyclic chemistry. 3rd ed. Oxford (UK): Elsevier; 2010. 
[4] Pozharskii AF, Katritzky AR, Soldatenkov AT. Heterocycles in life and society: an introduction to heterocyclic chemistry, biochemistry and applications. 2nd ed. Chichester (UK): Wiley; 2011.

[5] Chambers RD. Fluorine in organic chemistry. New York (NY): John Wiley \& Sons; 1973.

[6] Ishikawa N, Kobayashi Y. Fusso Kagoubustu (Compounds of fluorine). Tokyo (Japan): Kodansha; 1979.

[7] Kirk KL. Biochemistry of halogenated organic compounds. New York (NY): Plenum Press; 1991; Chapter 3, pp. 65-103 and Chapter 5, pp. 145-150.

[8] Kitazume T, Ishihara T, Taguchi T. Fusso No Kagaku (Chemistry of fluorine). Tokyo (Japan): Kodansha Scientific; 1993; Chapter 5, pp. 151-192.

[9] Filler R, Naqvi SM. Biomedical aspects of fluorine chemistry. In: Filler, R., Kobayashi, Y., editors. Amsterdam (the Netherlands): Elsevier; 1993, pp. 1-32.

[10] Banks RE, Smart BE, Tatlow JC. Organofluorine chemistry: principles and commercial applications. New York (NY): Plenum Press; 1994.

[11] Kukhar VP, Soloshonok VA, Eds. Fluorine-containing amino acids, synthesis and properties. Chichester (UK): John Wiley \& Sons; 1995.

[12] Hiyama, T, Eds. Organofluorine compounds. Chemistry and applications. New York (NY): Springer; 2000.

[13] McCarthy JR. Utility of fluorine in biologically active molecules, ACS fluorine division tutorial, 219th National ACS Meeting. San Francisco (CA), March 26; 2000.

[14] McCarthy JR. Fluorine in Drug Design: A Tutorial Review. 17th Winter Fluorine Conference, St. Pete Beach (FL); 2005.

[15] Thayer AM. Rapid screening and optimization of enzymatic activity, along with available, easy-to-use enzymes, are making biocatalysis a handy tool for chiral synthesis. Chem Eng News. 2006;84(33):15-25. doi:10.1021/cen-v084n033.p015

[16] Bégué J-P, Bonnet-Delpon D. Bioorganic and medicinal chemistry of fluorine. Hoboken (NJ): Wiley; 2007.

[17] Kirk KL. Fluorination in medicinal chemistry: methods, strategies, and recent developments. Org Process Res Dev. 2008;12 (2):305-21. doi:10.1021/op700134j

[18] Ojima I. Fluorine in medicinal chemistry and chemical biology. Oxford (UK): Blackwell; 2009.

[19] Kirsch P. Modern fluoroorganic chemistry. Weinheim (Germany): Wiley-VCH; 2013.

[20] Wang J, Sánchez-Roselló M, Aceña JL, del Pozo C, Sorochinsky AE, Fustero S, Soloshonok VA, Liu H. Fluorine in pharmaceutical industry: fluorine-containing drugs introduced to the market in the last decade (2001-2011). Chem Rev. 2014;114(4):2432506. doi:10.1021/cr4002879

[21] Welch JT. Eswarakrishman S. Fluorine in bioorganic chemistry. New York (NY): Wiley; 1991.

[22] Filler R, Kobayashi Y, Yagupolskii LM. Organofluorine compounds in medicinal chemistry and biomedical applications. Amsterdam, New York (NY): Elsevier; 1993.

[23] Petrov VA. Fluorinated heterocyclic compounds: synthesis chemistry, and applications. Hoboken (NJ): Wiley; 2009.

[24] Welch JT. Tetrahedron report number 221: advances in the preparation of biologically active organofluorine compounds. Tetrahedron. 1987;43(14):3123-97. doi:10.1016/S0040-4020 (01)90286-8

[25] Silvester MJ. Recent advances in fluoroheterocyclic chemistry. Adv Heterocycl Chem. 1994;59:1-38. doi:10.1016/S0065-2725 (08)60006-9

[26] Lin P, Jiang J. Synthesis of monotrifluoromethyl-substituted saturated cycles. Tetrahedron. 2000;56(23):3635-71. doi:10. 1016/S0040-4020(00)00158-7
[27] Erian AW. Recent trends in the chemistry of fluorinated five and six-membered heterocycles. J Heterocycl Chem. 2001;38 (4):793-808. doi:10.1002/jhet.5570380401

[28] Muzalevskiy VM, Shastin AV, Balenkova ES, Haufe G, Nenajdenko VG. Synthesis. 2009;23:3905-29.

[29] Prakash GKS, Mandal M, Schweizer S, Petasis NA, Olah GA. Stereoselective Synthesis of anti- $\alpha$-(difluoromethyl)- $\beta$-amino alcohols by boronic acid based three-component condensation. Stereoselective preparation of (2S,3R)-difluorothreonine. J Org Chem. 2002;67(11):3718-23. doi:10.1021/jo011116w

[30] Narjesa F, Koehlera KF, Kocha U, Gerlacha B, Colarussoa S, Steinkühlerb C, Brunettib M, Altamurab S, De Francescob R, Matassaa VG. A designed P1 cysteine mimetic for covalent and non-covalent inhibitors of HCV NS3 protease. Bioorg Med Chem Lett. 2002;12(4):701-4. doi:10.1016/S0960-894X(01)00842-3

[31] Erickson JA, McLoughlin JI. Hydrogen bond donor properties of the difluoromethyl group. J Org Chem. 1995;60(6):1626-31. doi:10.1021/jo00111a021

[32] Li Y, Hu J. Facile synthesis of chiral $\alpha$-difluoromethyl amines from N-(tert-butylsulfinyl) aldimines. Angew Chem. 2005;117 (36):6032-6.

[33] Li Y, Hu J. Facile synthesis of chiral $\alpha$-difluoromethyl amines from N-(tert-butylsulfinyl) aldimines. Angew Chem Int Ed. 2005;44(36):5882-6. doi:10.1002/anie.200501769

[34] Prakash GKS, Weber C, Chacko S, Olah GA. New electrophilic difluoromethylating reagent. Org Lett. 2007;9(10):1863-6. doi:10.1021/ol070195g

[35] Rohdich N, Roepke RKA, Zschiesche E. A randomized, blinded, controlled and multi-centered field study comparing the efficacy and safety of Bravecto ${ }^{\mathrm{TM}}$ (fluralaner) against Frontline ${ }^{\mathrm{TM}}$ (fipronil) in flea- and tick-infested dogs. Parasit Vectors. 2014;7 (1):83. doi:10.1016/j.vetpar.2006.10.028

[36] Quan ML, Ellis CD, Liauw AY, Alexander RS, Knabb RM, Lam G, Wright MR, Wong PC, Wexler RR. Design and synthesis of isoxazoline derivatives as factor Xa inhibitors. J Med Chem. 1999;42 (15):2760-73. doi:10.1021/jm980406a

[37] Kumar V, Aggarwal R, Singh SP. The reaction of hydroxylamine with aryl trifluoromethyl- $\beta$-diketones: synthesis of 5-hydroxy5 -trifluoromethyl- $\Delta 2$-isoxazolines and their dehydration to 5trifluoromethylisoxazoles. J Fluorine Chem. 2006;127(7):880-8. doi:10.1016/j.jluchem.2006.03.009

[38] Bravo P, Bruché L, Crucianelli M, Farina A, Meille SV, Merli A, Seresini P. ChemInform abstract: asymmetric 1,3-dipolar cycloadditions of nitrile oxides and nitrones with fluorosubstituted chiral vinyl sulfoxides. J Chem Res Synop. 1996; 27(52) 348-9. doi:10.1002/chin.199652150

[39] Kawai H, Tachi K, Tokunaga E, Shiro M, Shibata N. Trifluoromethylation of aromatic isoxazoles: regio- and diastereoselective route to 5-Trifluoromethyl-2-isoxazolines. Angew Chem. 2011;123(34):7949-52. doi:10.1002/ange.201102442

[40] Kawai H, Tachi K, Tokunaga E, Shiro M, Shibata N. Trifluoromethylation of aromatic isoxazoles: regio- and diastereoselective route to 5-trifluoromethyl-2-isoxazolines. Angew Chem Int Ed. 2011;50(34):7803-06. doi:10.1002/anie.201102442

[41] Kawai H, Sugita Y, Tokunaga E, Sato H, Shiro M, Shibata N. Diastereoselective additive trifluoromethylation/halogenation of isoxazole triflones: synthesis of all-carbon-functionalized trifluoromethyl Isoxazoline Triflones. Chemistry Open. 2014; 3(1):14-18. doi:10.1002/open.201300044

[42] More than 27000 compounds of isoxazolines have been registered in SciFinder database in November 2014, and most of them have been protected by over 170 patents.

[43] Only 19 compounds of isoxazolines have been registered in SciFinder database in November 2014, and they have been protected by over 4 patents. 
[44] Takeshi M, Takamasa K, Takashi M, Manabu Y, Mitsuaki K. US20070066617, W02005085216, 2005.

[45] Takeshi M, Takamasa K, Takashi M, Manabu Y, Mitsuaki K. US20070066617, 2007.

[46] Jyun I, Masahiro K, WO2009022746, 2009.

[47] Yves CJ, Peter R, Thomas P, Myriem E. Q. W0201302 6931, 2013.

[48] Hagiwara T, Fuchikami T. Difluoroalkylation of carbonyl compounds with (1,1-difluoroalkyl) silane derivatives. Synlett. 1995; 1995(7):717-8. doi:10.1055/s-1995-5070

[49] Zhao Y, Huang W, Zheng J, Hu J. Efficient and direct nucleophilic difluoromethylation of carbonyl compounds and imines with Me3SiCF2H at ambient or low temperature. Org Lett. 2011;13 (19):5342-5. doi:10.1021/ol202208b

[50] Donati D, Fiorenza M, Moschi E, Sarti-Fantoni P. Photodimerization of 3-methyl-4-nitro-5-styrylisoxazole in the solid state. J Heterocycl Chem. 1977;14(5):951-51. doi:10.1002/jhet.5570 140549

[51] Donati D, Fiorenza M, Sarti-Fantoni P. Photochemical reactions of 3-methyl-4-nilro-5-styrylisoxazole in solution, in the solid slate and adsorbed on silica gel. J Heterocycl Chem. 1979;16 (2):253-6. doi:doi10.1002/jhet.5570160212

[52] Sarti-Fantoni P, Donati D, De Sio F, Moneti G. Alkaline hydrolysis of 3-methyl-4-nitro-5-styrylisoxazole with $\mathrm{Na}^{18} \mathrm{OH}$. J Heterocycl Chem. 1980;17(7):1643-4. doi:10.1002/jhet. 5570170764

[53] Chimichi S, De Sio F, Donati D, Fina G, Pepino R, Sarti-Fantoni P. The preparation of coumaric acids via styrylisoxazoles. Heterocycles. 1983;20:263-7. doi:10.3987/R-1983-02-0263

[54] Baracchi A, Chimichi S, De Sio F, Donati D, Nesi R, Sarti-Fantoni P, Torroba T. Preparation of ${ }^{18}$ O-bilabelled carboxylic acids: cinnahic and phenylpropiolic acids via styrylisoxazoles. J Labelled Compd Radiopharm. 1986;23(5):487-493. doi:10.1002/jlcr. 2580230505

[55] Baracchi A, Chimichi S, De Sio F, Donati D, Nesi R, Sarti-Fantoni P, Torroba T. Preparation of chloro- $\alpha$-truxillic acids via 3methyl-4-nitro-5-styrylisoxazole photodimers. Heterocycles. 1986;24(10):2863-70. doi:10.3987/R-1986-10-2863

[56] Adamo MFA, Duffy EF. Multicomponent synthesis of 3-heteroarylpropionic acids. Org Lett. 2006;8(22):5157-9. doi:10.1021/ ol062151y

[57] Adamo MFA, Konda VR. Multicomponent synthesis of 3indolepropionic acids. Org Lett. 2007;9(2):303-5. doi:10.1021/ ol0627698

[58] Adamo MFA, Duffy EF, Donati D, Sarti-Fantoni P. Modular synthesis of isoxazolopyridones and pyrazolopyridones. Tetrahedron. 2007;63(9):2047-52. doi:10.1016/j.tet.2006.12.044

[59] Adamo MFA, Duffy EF, Donati D, Sarti-Fantoni P. Modular syntheses of isoxazoloazepinones and pyrazoloazepinones. Tetrahedron. 2007;63:2684-8. doi:10.1016/j.tet.2007.01.018

[60] Adamo MFA, Konda VR, Donati D, Sarti-Fantonic P, Torroba T. Three multicomponent reactions of 3,5-dimethyl-4-nitroisoxazole. Tetrahedron. 2007;63(39):9741-5. doi:10.1016/j.tet.2007.07. 015
[61] Adamo MFA, Nagabelli M. Synthesis of homochiral dihydroxy-4nitroisoxazolines via one-pot asymmetric dihydroxylation-reduction. Org Lett. 2008;10(9):1807-10. doi:10.1021/ ol800397z

[62] Adamo MFA., Donati D, Sarti-Fantoni P, Buccioni A. Practical route for N,O-heteroatom interchange in 3,5-disubstituted4-nitroisoxazoles. Tetrahedron Lett. 2008;49(6):941-4. doi:10. 1016/j.tetlet.2007.12.019

[63] Adamo MFA, Konda VR. A multicomponent synthesis of cyclopropanes. Tetrahedron Lett. 2008;49(43):6224-6. doi:10.1016/ j.tetlet.2008.08.033

[64] Adamo MFA, Bruschi S, Suresh S, Piras L. Aziridination of 3methyl-4-nitro-5-styrylisoxazoles. Tetrahedron Lett. 2008;49(52), 7406-9. doi:10.1016/j.tetlet.2008.10.061

[65] Baschieri A, Bernardi L, Ricci A, Suresh S, Adamo M. F. A. Catalytic asymmetric conjugate addition of nitroalkanes to 4-nitro-5-styrylisoxazoles. Angew Chem. 2009;121(49):9506-9. doi:10.1002/anie.200905018

[66] Baschieri A, Bernardi L, Ricci A, Suresh S, Adamo MFA. Catalytic asymmetric conjugate addition of nitroalkanes to 4-nitro-5-styrylisoxazoles. Angew Chem Int Ed. 2009;48(49):9342-5. doi:10. 1002/anie.200905018

[67] Kawai H, Sugita Y, Tokunaga E, Shibata N. Synthesis of isoxazole triflones. Eur J Org Chem. 2012;2012(7):1295-8. doi:10.1002/ ejoc.201101814

\section{COMPETING INTERESTS}

The authors declare no competing interests.

\section{PUBLISHING NOTES}

(C) 2014 Xin Wang et al. This work has been published open access under Creative Commons Attribution License CC BY 4.0, which permits unrestricted use, distribution, and reproduction in any medium, provided the original work is properly cited. Conditions, terms of use and publishing policy can be found at www.scienceopen.com.

Please note that this article may not have been peer reviewed yet and is under continuous post-publication peer review. For the current reviewing status please click here or scan the QR code on the right.

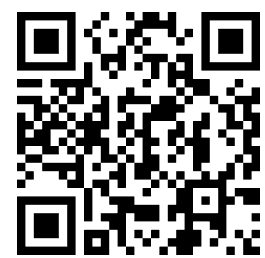

\section{scienceOPEN.com}

research+publishing network 